\title{
Wall-type and indoor residual spraying application quality affect the residual efficacy of indoor residual spray against wild malaria vector in southwest Ethiopia
}

Zerihun Desalegn (10, Teklu Wegayehu and Fekadu Massebo*

\begin{abstract}
Background: Residual efficacy of indoor residual spray may vary with different spray quality and wall surfaces types. This study evaluated the impact of spray quality and wall surface types on residual efficacy of propoxur against wild Anopheles gambiae sensu lato (s.l.) in southwest Ethiopia.

Methods: Thirty houses of different mud wall surfaces (10 smooth, 10 rough, 10 painted) were selected and randomly allocated into routine and standard spray. The routine spray was conducted by district health office as usual, while the standard spray was done by strictly following guidelines. Three control houses were selected from unsprayed nearby semi-urban. Wild An. gambiae s.l. were used for wall bioassay tests. Two-way mixed model analysis of variance was used to analyse the data. The mean variation between wall and spray types was compared by post hoc analysis of IBM SPSS version 20.

Results: On standard spray, knockdown rate was $95.3 \%$ on painted, $82 \%$ on smooth and $72.5 \%$ on rough surface at week 17 of post-spray, whereas on routine spray it was $82.7 \%$ on painted, $48.7 \%$ on smooth and $60 \%$ on rough surface. On standard spray, mortality rate of An. gambiae s.l. was $99.3 \%$ on painted surface, $90 \%$ on smooth and $80 \%$ on rough surface. On routine spray, it was $89.3 \%$ on painted, $61.3 \%$ on smooth and $65 \%$ on rough surface at week 17 of post-spray. The painted wall surface showed the highest knockdown rate (86.4-100\%) on standard and (73.8-91.5\%) routine spray; mortality rate was more than $80 \%$ on both spray types during the 17 weeks of follow-up regardless of spray types. The lower mortality rate and residual effect was observed on routine smooth and rough wall surfaces. The residual efficacy of propoxur was $>80 \%$ at week 17 on standard spray regardless of the wall types and it was $<80 \%$ on routine spray except painted wall surface.
\end{abstract}

Conclusion: The painted wall surface and standard spray showed better residual efficacy. Therefore, it is recommended to consider the wall surface available in the community to estimate the residual lifespan of the insecticide, and strictly to follow the spray guideline to improve the effectiveness of indoor residual spray.

Keywords: Propoxur, Routine spray, Standard spray, Wall surface type

*Correspondence: fekadu.massebo@amu.edu.et

Department of Biology, Arba Minch University, Arba Minch, Ethiopia 


\section{Background}

Malaria is spread from one person to another by female mosquitoes of the genus Anopheles and is one of the major public health problems of the world with an annual estimate of 212 million cases and 429,000 deaths, mainly in the sub-Saharan Africa, in 2015 [1]. It was accountable for about 303,000 malaria deaths in under-five children globally in 2015, and 10\% of under-five deaths in subSaharan Africa. On the other hand, malaria case incidence decreased by $41 \%$ and mortality by $62 \%$ globally between 2000 and 2015 [1].

Long-lasting, insecticide-treated nets (LLINs) and indoor residual spray (IRS) are the two cornerstone malaria control interventions contributing to current malaria reduction [2]. The two intervention tools are effective against indoor resting and biting malaria vectors [3]. In the 1950s, dichlorodiphenyltrichloroethane (DDT)-based IRS was initiated and implemented to control malaria in Ethiopia [4]. It was the principal tool in the 1950s' and 1960s' malaria eradication programme. Its use was discontinued in 2009 and was replaced by pyrethroid insecticides, due to the widespread distribution of DDTresistant malaria vectors [5]. In 2011, pyrethroid-based IRS was replaced by carbamate, due to the high level of resistance in malaria vector populations. Currently, carbamate insecticides are in use for IRS and the principal malaria vector, Anopheles arabiensis is susceptible to these insecticides in most parts of the country $[5,6]$.

The insecticide sprayed on the wall surface should be stable in order to minimize the number of spraying cycle in the targeted malaria transmission seasons. In areas where the transmission season is more than 6 months, multiple spraying can be required and become expensive due to the high demands of logistics [7]. However, residual efficacy of IRS may be varied with wall type and the quality of spray application $[8,9]$. Moreover, the effectiveness of IRS depends on resistance status of malaria vectors and its residual time on the wall surface [9]. Poor quality application may contribute to insecticide resistance and could be a challenge for malaria vector control [10]. If the residual effect of IRS is shorter than expected, it may contribute to an increase in incidence of malaria infection where the malaria transmission season exceeds the residual effect of insecticides [11].

There are different wall surfaces in the study area and it would be useful to determine the residual life of insecticide on different wall surface. There is little information on the effect of wall types and application quality on the residual efficacy of carbamate insecticides used for IRS in Ethiopia [12]. Therefore, the impact of wall type and application quality on the efficacy of propoxur was assessed against the principal malaria vector, which might help to modify the IRS implementation programme.

\section{Methods \\ Description of the study area}

Gamo Gofa is one of 13 zones in the Southern Nations Nationalities and Peoples Region (SNNPR). Arba Minch, the capital of Gamo Gofa, is located $505 \mathrm{~km}$ southwest of Addis Ababa and $275 \mathrm{~km}$ from Hawassa, the regional capital city. Malaria is one of the public health problems in the Arba Minch Zuria district. Shellie Mella is among the 11 malarious villages in the district. This village was selected purposely, based on its malaria endemicity and accessibility for study. It is located $20 \mathrm{~km}$ south of Arba Minch at an altitude of 1120-1380 m above sea level (masl). The annual rainfall is $900-1300 \mathrm{~mm}$ and its annual temperature is $25-36{ }^{\circ} \mathrm{C}$. The total population of the village was 10,721 (5253 males and 5468 females) within 2188 households. The main source of income is agriculture (banana and mango cultivation).

The population lives in houses with different wall surfaces, including rough, painted or smooth mud walls with grass thatched or iron roofs. There is one governmental health centre and one health post in the study area. IRS and LLINs are the two major vector control interventions implemented by the District Health Office. The coverage of the IRS was $98 \%$ and that of LLINs was $99 \%$ in 2016.

\section{Study design}

This experimental trial was carried out in Shellie Mella from August to November 2016. The list of houses was taken from health post and 30 houses were selected randomly from the community with different wall types (10 smooth, 10 painted, 10 rough mud walls). The houses were then coded and assigned randomly into two arms before spraying. Fifteen houses (five from each wall type) were assigned for standard spray and the other 15 houses (five from each wall type) for routine spray. The housing condition of the two groups was characterized to make sure that the houses are comparable in most characteristics other than application type to minimize bias. Three control houses with the three wall types (rough, painted, smooth mud wall) and comparable characteristics to the intervention houses were selected from unsprayed nearby semi-urban village. Control houses were used for adjustment of percentage mortality of mosquito by Abbott's formula [13]. Spray operators, supervisors and data collectors were blinded to spray type allocation and bioassay test to minimize biases. Verbal and written consent was obtained from head of households before spraying. The routine spray was coordinated by District Health Office and conducted as usual by spray operators. Standard 
spray was conducted by strictly following the WHO and national spray guideline using the same spray operators.

\section{Test mosquito rearing procedure}

The larvae and pupae of Anopheles mosquitoes were collected from Kulfo River and surrounding areas and then transported to Arba Minch University Medical Entomology laboratory. Pupae were separated using pipette and placed into adult mosquito cages. Adults were provided with cotton wool soaked in $10 \%$ sugar solution. The insectary environment was maintained at temperature of $27 \pm 2{ }^{\circ} \mathrm{C}$ and relative humidity of $60 \pm 10 \%$. Then, 3-5 days old sugar-fed female Anopheles gambiae s.l. were used for wall bioassay tests.

\section{Insecticide susceptibility tests}

Insecticide susceptibility test was carried out in the laboratory against the wild female An. gambiae s.l. using WHO test kit and $0.1 \%$ propoxur-impregnated paper. This test was done to ensure susceptibility of the species before conducting cone bioassay tests. The procedure was carried out according to WHO guidelines [13]. Four replicates of 25 female mosquitoes were exposed to insecticide-impregnated test paper in each tube for $1 \mathrm{~h}$. Two replicates of the same batch of mosquito were exposed on oil-impregnated papers for control. Mortality was recorded after $24 \mathrm{~h}$ of exposure. The resistance status of An. gambiae s.l. was determined according to the latest WHO [13] criteria as follows:

- Mortality rates between 98 and 100\% indicate full susceptibility.

- Mortality rates between 90 and $97 \%$ require further investigation.

- Mortality rates $<90 \%$, the population considered resistant to the tested insecticide [13].

Anopheles gambiae s.l. were fully susceptible to propoxur $0.1 \%$ with $100 \%$ mortality after $24 \mathrm{~h}$.

\section{Inclusion and exclusion criteria}

All houses with smooth, rough and painted mud walls were eligible for the study. Householders who declined informed consent, newly constructed houses and where mud walls were not dry during the selection were excluded. Houses with humans and animals living together were excluded as it is unusual to share houses with animals in the area. Moreover, houses were excluded if kitchen and living room were one to minimize the impact of smoking on insecticide.

\section{Indoor residual spraying application}

\section{Routine and standard spraying application}

Those houses selected and coded for routine spray were sprayed by trained community spray operators during the routine spray schedule of the area. The spray was coordinated by, and training given for 6 days to spray operators by District Health Office. Propoxur 50\% water-dispersible powder (WP) available under trade name of FICAM $^{\circledR}$ was supplied by District Health Office.

The other 15 houses ( 5 smooth, 5 painted, 5 rough mud walls) randomly selected for the standard spray were sprayed with the same chemical deployed in the community. Two local spray operators from those involved in routine spray were used for standard spray. The difference was that in standard spray the operators strictly followed the WHO and national spraying operation guidelines [9, 14]. Spraying was conducted in collaboration with District Health Office. Additional orientation on standard spraying operation was given by IRS experts from the District Health Office. Spray equipment, personnel protective clothing, goggles, gloves and other materials for safety of spray operators were obtained from District Health Office. The insecticide application was done at rate of $2 \mathrm{~g} / \mathrm{sq} \mathrm{m}$ in the form of a WP [15] using a Hudson $\mathrm{X}$-pert ${ }^{\circledR}$ sprayer (8-1 capacity) with HSS-8002E nozzle, which was equipped with a regulator adjusted at angle of $80^{\circ}$ and discharging rate of $760 \mathrm{~m}$ per minute at a standard tank pressure of $55 \mathrm{psi}$.

The spray operators strictly followed the instructions on the product label to ensure safe and correct mixing (one sachet or $500 \mathrm{~g}$ in 81 water), handling and application of insecticides. The insecticide was mixed outdoors in well ventilated areas [9]. Before starting spraying, information was provided for householders about the safety, purpose and time of spraying. The inhabitants were requested to prepare and remove all materials such as water, food and cooking utensils from the houses before spraying. Household members were allowed to enter the house $2 \mathrm{~h}$ after spraying. The inhabitants were also requested not to wash, paint or re-plaster the sprayed walls [9]. Spray operators also reassured householders about the safety of insecticide being used, and that applied insecticides did not damage walls, ceilings and furniture.

\section{Cone bioassay test using wild Anopheles gambiae s.l.}

The wall bioassay tests were carried out by using the standard WHO cone method. The first bioassay was carried out after 1 day of spraying, then after 1 week and then every 4 weeks for 4 months from August to November 2016. Each day, both spray types were conducted on 15 houses of three wall surfaces. Three WHO cones with 12-cm diameter were firmly fixed on each wall surface for 
both standard and routine sprayed houses at three different locations (lower, middle and upper) above the ground [9].

Then, 10 3-5 days old female An. gambiae s.l. were introduced into each cone fixed on the wall by using mouth aspirator. At the same day, separate cones were fixed on control houses and the same number of mosquitoes was introduced using separate mouth aspirator to avoid contamination. After $30 \mathrm{~min}$ of exposure, the mosquitoes were transferred into plastic cups and $10 \%$ sugar was provided. The temperature was maintained at $27 \pm 2{ }^{\circ} \mathrm{C}$ and relative humidity was $70 \pm 10 \%$ for $24 \mathrm{~h}$. The percentage of knockdown after $1 \mathrm{~h}$ of exposure was recorded. Mortality rate was recorded after a 24-h holding period.

\section{Safety procedures}

On the day of spraying all family members were advised to remain out of rooms for $2 \mathrm{~h}$ after spraying to avoid any possible risk during and after the spraying of their houses. The adults of household were also advised to tell their children not to touch the sprayed walls for at least 1 day after spraying. For environmental safety, the containers or sachets were returned to District Health Office for proper disposal [9]. Both spray operators and household members were informed of signs of adverse effect and advised to report any adverse effect of insecticide during 1 week post-spraying and no adverse effect was reported from either household members or spray operators during spray time and 1 week after spraying.

\section{Outcome variables}

The first primary outcome variable was the time to knockdown of An. gambiae s.l. within 30 min of exposure on different wall surfaces and spray types. The second primary outcome variable was the percentage the mortality rate after $24 \mathrm{~h}$ holding period during the follow-up period.

\section{Data analysis}

Knockdown and mortality rates were calculated and analysed according to $\mathrm{WHO}$ protocol to determine the efficacy of IRS. Percentage of knockdown was calculated after $30 \mathrm{~min}$ exposure. The percentage mortality was calculated after $24 \mathrm{~h}$ holding period. All mosquitoes that could fly were considered alive. Control mortality was less than $5 \%$ in all bioassay during the study period and hence was not corrected by Abbott's formula.

Two-way mixed model ANOVA was used to determine the mean knockdown and mortality variation among the wall surfaces and spray types. Post-hoc test analysis was employed to identify spray type and wall type with significant difference. A significance test was done by $p$ value $<0.05$. Treatment was considered effective until mortality rate in exposed mosquitoes was $\geq 80 \%$ within $24 \mathrm{~h}$ [16]. Two houses (one standard and one routine rough wall surfaces) were excluded from analysis as they were replastered. Data were entered and analysed using IBM SPSS version 20 (SPSS Inc, Chicago, USA).

\section{Results}

Knockdown rate on different spray and wall types

The knockdown rates of An. gambiae s.l. after $1 \mathrm{~h}$ exposure varied on standard and routine spray of different wall surfaces (Tables 1 and 2). The knockdown rate was $100 \%$ for all wall surfaces in both spray types on the first week of bioassay. On both spray types, painted wall surface revealed $100 \%$ knockdown rate for 13 weeks and more than $80 \%$ at week 17 , while it was low on smooth and rough wall surfaces of both spray types. At week 17 of the standard spray, the knockdown rate was $95.3 \%$ (95\% confidence interval (CI) 86.4-100) on painted, $82 \%$ (95\% CI 73-90.9) on smooth, and $72.5 \%$ (95\% CI 62.6-82) on rough surface. Whereas on routine spray, the knockdown rate was $82.7 \%$ (95\% CI 73.8-91.5) on painted, $48.7 \%$ (95\% CI 39.8-57.5) on smooth, and $60 \%$ (95\% CI 50-69.9\%) on rough surface.

The mean knockdown rate of mosquito was significantly different among spray types applied $(\mathrm{F}(1,78)=44.6$, $\mathrm{P}<0.001)$. Also it was different among different wall surfaces sprayed $(\mathrm{F}(2,78)=32.5, \mathrm{P}<0.001)$. The significant difference occurred between standard painted and standard rough wall surface $(\mathrm{P}<0.001)$ (Table 3$)$ and standard smooth and rough starting week $13(\mathrm{P}<0.0001)$ (Table 1$)$.

Table 1 Mean mosquito knockdown rate on different wall surface by standard spray through time post spray in Shellie Mella, southwest Ethiopia

\begin{tabular}{lllllll}
\hline Wall types & \multicolumn{2}{l}{ Time in weeks } & & & & \\
\cline { 2 - 7 } & Day $\mathbf{1}$ & Week $\mathbf{1}$ & Week $\mathbf{5}$ & Week $\mathbf{9}$ & Week 13* & Week 17* \\
\hline Painted & 100 & 100 & 100 & 100 & 100 & 95.3 \\
Smooth & 100 & 100 & 100 & 98.7 & 99.3 & 82 \\
Rough & 100 & 100 & 96.7 & 87.5 & 78.3 & 72.5 \\
\hline
\end{tabular}

* Significant difference $(P<0.05)$ 
There was no significant difference between standard painted and standard smooth throughout the follow-up period $(P=0.9)$. However, on routine spray, the significant difference occurred between painted and smooth, and painted and rough starting week $5 \quad(\mathrm{P}<0.0001)$ (Table 2). But, there was no difference between rough and smooth throughout the follow-up period $(\mathrm{P}>0.05)$ on routine spray. The post hoc test analysis showed that the highest knockdown rate was observed on painted wall surface irrespective of type of spraying (83-100\%). However, the knockdown rate was not varied in painted wall surface ranging between 95 and $100 \%$ on standard and $83-100 \%$ on routine spray $(\mathrm{P}=0.37)$. In the control houses there was no mosquito knockdown observed after $1 \mathrm{~h}$ of exposure in all wall surfaces.

\section{Mortality rate on different spray and wall types}

The mortality rate of An. gambiae s.l. after $24 \mathrm{~h}$ holding time on different wall types by standard and routine spray application is indicated in Tables 4, 5. More than $80 \%$ mortality rate was observed on standard spray on all wall types during the 17 weeks bioassay tests. On routine spray, it gradually decreased from 100 to $61 \%$ on smooth and 100 to $65 \%$ on rough wall types. The mortality rate of An. gambiae s.l. was above $80 \%$ on painted wall even in routine spray at 17 weeks.

There was a significant difference in mean mortality rate of $A n$. gambiae s.l. between spray types $(\mathrm{F}(1,78)=58.27, \mathrm{P}<0.001)$. Standard spray showed better mosquito mortality rate than routine spray during 17 weeks of assessment on all three wall types. There was also significant difference in the mean mortality rate between the wall types $(F(2,78)=26, P<0.001)$. Posthoc test showed that the highest mortality rate was on painted mud wall surface. On standard spray, the difference on mortality rate was observed only between painted and rough surface at week $17(\mathrm{P}<0.001)$, but no difference was seen between painted and smooth wall

Table 2 Mean mosquito knockdown rate on different wall types by routine spray through time post spray in Shellie Mella, southwest Ethiopia

\begin{tabular}{|c|c|c|c|c|c|c|}
\hline \multirow[t]{2}{*}{ Wall types } & \multicolumn{6}{|c|}{ Time in weeks } \\
\hline & Day 1 & Week 1 & Week $5^{*}$ & Week $9 *$ & Week 13* & Week $17^{*}$ \\
\hline Painted & 100 & 100 & 100 & 100 & 100 & 82.7 \\
\hline Smooth & 100 & 100 & 84 & 71.3 & 82 & 48.7 \\
\hline Rough & 100 & 100 & 87.5 & 78.3 & 68.3 & 60 \\
\hline
\end{tabular}

* Significant difference $(\mathrm{P}<0.05)$

Table 3 Mean mosquito knockdown rate on wall types and spray type at 17 weeks bioassay in Shellie Mella, southwest Ethiopia

\begin{tabular}{|c|c|c|c|c|c|c|}
\hline Wall type & Spray type & $\mathbf{N}$ & Mean \% knockdown & $95 \% \mathrm{Cl}$ & SE & P-value \\
\hline \multirow[t]{2}{*}{ Painted } & Standard & 15 & $95.3^{\mathrm{a}}$ & $86-100$ & 4.5 & 0.37 \\
\hline & Routine & 15 & $82.7^{\mathrm{a}}$ & 72.9-91 & 4.5 & \\
\hline \multirow[t]{2}{*}{ Smooth } & Standard & 15 & $82^{\mathrm{a}}$ & $73.1-90.9$ & 4.5 & $<0.001$ \\
\hline & Routine & 15 & $48.7^{b}$ & $39.8-57.7$ & 4.5 & \\
\hline \multirow[t]{2}{*}{ Rough } & Standard & 12 & $67.5^{c}$ & $58-76.4$ & 4.9 & 0.9 \\
\hline & Routine & 12 & $64.4^{b, c}$ & $52.7-76$ & 4.9 & \\
\hline
\end{tabular}

Change in letters between columns indicates statistically significant difference between wall and spray types

Table 4 Mean mortality rates of mosquito on different wall surface by routine spray in Shellie Mella, southwest Ethiopia

\begin{tabular}{lllllll}
\hline Wall types & \multicolumn{2}{l}{ Time in weeks } & & & & \\
\cline { 2 - 7 } & Day $\mathbf{1}$ & Week $\mathbf{1}$ & Week & Week & Week 13* & Week 17* \\
\hline Painted & 100 & 100 & 100 & 100 & 100 & 89.3 \\
Smooth & 100 & 100 & 98.7 & 82.3 & 82 & 61.3 \\
Rough & 100 & 100 & 100 & 91.7 & 87.5 & 65
\end{tabular}

* Indicates significant difference $(P<0.05)$ 
types (Table 6). On routine spray, the significant difference was occurred from week 9 (Tables 4, 5). However, mortality rate was not significantly varied between smooth and rough mud wall types on both spray types $(P=0.83)$. Also, mortality rate was not significantly varied on painted wall surface on standard (92.8-100\%) and routine $(82.8-95.8 \%)$ spray types $(\mathrm{P}=0.84)$. In the control houses, mosquito mortality rate after $24 \mathrm{~h}$ recovery period was less than $5 \%$ in all wall types.
Residual time of propoxur on different spray surfaces and wall types

The residual effect of propoxur was defined by mortality rate based on the WHO cut-off point which was $>80 \%$. The mortality rate of $A n$. gambiae s.l. was $>80 \%$ in both spray and wall types until week 13 . However, the mortality rate on routine smooth and rough surface was $<80 \%$ at week 17 (Fig. 1). The residual time of propoxur was more than 17 weeks for standard spray irrespective of the wall

Table 5 Mean mortality rates of mosquito on different wall surface by standard spray in Shellie Mella, southwest Ethiopia

\begin{tabular}{lllllll}
\hline Wall types & \multicolumn{2}{l}{ Time in weeks } & & & \\
\cline { 2 - 7 } & Day $\mathbf{1}$ & Week 1 & Week 5 & Week 9 & Week 13 & Week 17* \\
\hline Painted & 100 & 100 & 100 & 100 & 100 & 99.3 \\
Smooth & 100 & 100 & 100 & 100 & 100 & 90 \\
Rough & 100 & 100 & 100 & 100 & 97.5 & 80 \\
\hline
\end{tabular}

* Indicates significant difference $(P<0.05)$

Table 6 Mean mortality rate of mosquito on different wall surface versus spray type at week 17 in Shellie Mella, southwest Ethiopia

\begin{tabular}{|c|c|c|c|c|c|c|}
\hline Wall type & Spray type & $\mathrm{N}$ & Mean \% mortality & $95 \% \mathrm{Cl}$ & SE & P-value \\
\hline \multirow[t]{2}{*}{ Painted } & Standard & 15 & $99.3^{\mathrm{a}}$ & $92.8-100$ & 0.93 & \multirow[t]{2}{*}{0.84} \\
\hline & Routine & 15 & $89.3^{\mathrm{a}}$ & $82.8-95.8$ & 0.93 & \\
\hline \multirow[t]{2}{*}{ Smooth } & Standard & 15 & $90^{\mathrm{a}, \mathrm{c}}$ & $83.5-96.5$ & 0.93 & \multirow[t]{2}{*}{$<0.001$} \\
\hline & Routine & 15 & $61.3^{\mathrm{b}}$ & $54.8-67.8$ & 0.93 & \\
\hline \multirow[t]{2}{*}{ Rough } & Standard & 12 & $80^{c}$ & $72.7-87.3$ & 1.04 & \multirow[t]{2}{*}{$<0.001$} \\
\hline & Routine & 12 & $65^{b}$ & $57.7-72.3$ & 1.04 & \\
\hline
\end{tabular}

Change in letters between columns indicates statistically significant difference between wall and spray types

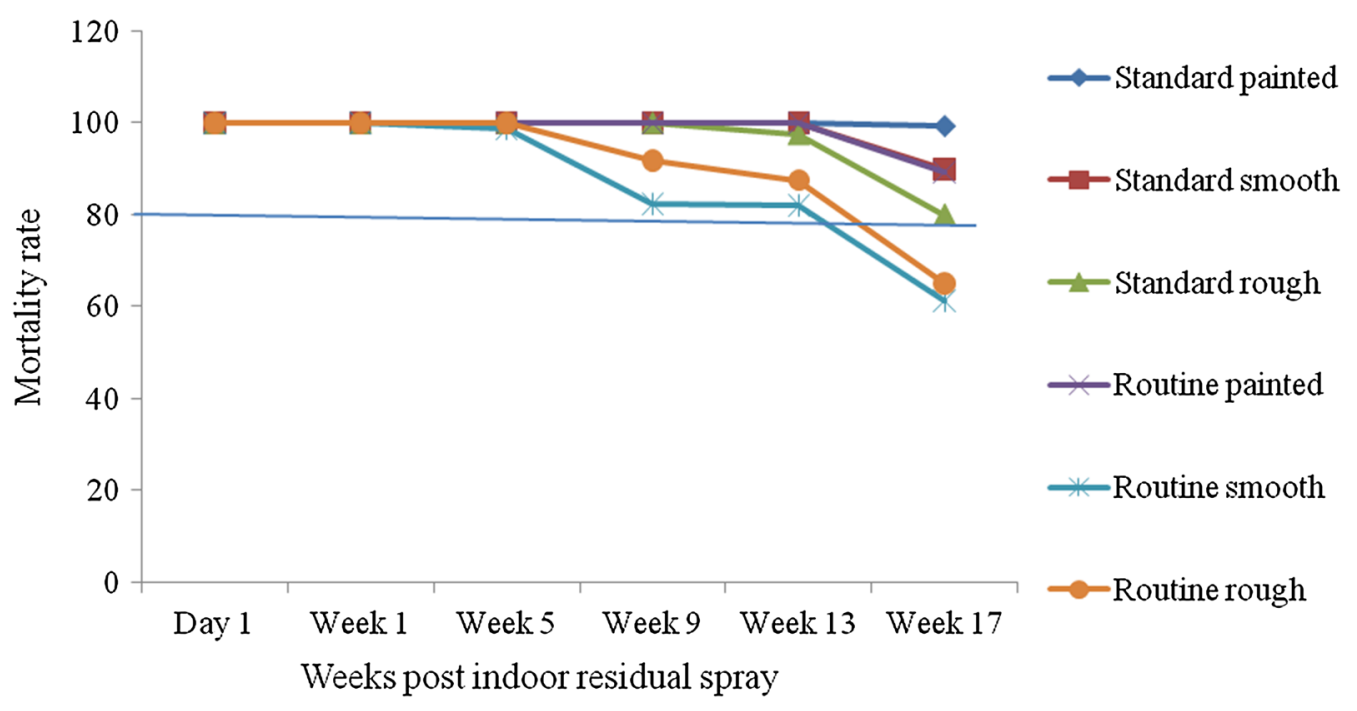

Fig. 1 WHO cut-off point (mortality rate $\geq 80 \%$ ) on different spray and wall types during the 17 weeks follow-up period in Shellie Mella, southwest Ethiopia 
types. In routine spray, the residual time was more than 17 weeks on painted wall surface only.

\section{Discussion}

This study indicated that the spray quality and wall types in the community affect the residual efficacy of IRS. The knockdown rate of Anopheles mosquito was varied in different spray type and wall surface. The knockdown rate was higher on standard painted wall, while it was lowest in routine smooth. The mortality rate of An. gambiae s.l. was high on standard painted wall and lowest on routine smooth. The residual duration of propoxur was influenced by spray and wall types. On standard spray, it was more than 17 weeks for all three wall types, while it was less than 17 weeks for routine spray except painted wall surface.

The standard spray and painted wall surface showed better knockdown rate. The experimental study conducted in Cameroon assessed the knockdown rate of An. gambiae sensu stricto (s.s.) exposed to bendiocarbtreated wood, concrete and mud wall surface [10]. They reported that more than $98 \%$ knockdown rate during 13 weeks observed on wood and concrete wall surface, while it was $80 \%$ on mud wall surface and there was statistically significant difference at $p<0.05$. Although the wall types used in that study were different from the present study, it showed that knockdown rate was affected by wall types. A study conducted in Karagwe district in Tanzania on residual effect of lambdacyhalothrin (capsule suspension) insecticide reported 100\% knockdown rate on painted surface in 3 months follow-up period, however, it was only $35 \%$ on mud surfaces [17]. Similarly in Muleba district, the highest knockdown rate was observed on painted surface (67\%) and the lowest on mud substrate (45\%) during 3 months follow-up [17]. This was consistent with the present study finding which showed the impact of wall type on knockdown rate and better knockdown rate observed on painted surfaces.

In current study, the standard spray showed better mortality rate on all wall surfaces. Also, painted wall surface has better effect on mortality of mosquito in both spray types. Better performance $(100 \%$ mortality rate) of bendiocarb sprayed on painted walls for up to 6 months was documented in Ethiopia, while the residual performance was significantly lower in dung and mud-plastered wall surfaces [18]. Another study in Adulala village in Ethiopia showed the highest mortality of mosquitoes on bendiocarb-sprayed, painted wall surface than others, but the difference was not significant [19]. The possible explanation for high performance of the painted wall surface for long periods could be due to the closing of small pores that pass insecticide through and may reduce the biodegradability of insecticide on walls. On the other hand, smooth and rough mud walls also have pores that may pass insecticide and may reduce efficacy of the insecticide.

Based on WHO recommendations [13], an ideal insecticide should have a minimum residual effect of greater or equal to $80 \%$ mosquito mortality after $24 \mathrm{~h}$ post-exposure during the recommended lifetime. In this finding the residual time of propoxur on standard spray was more than 17 weeks in all three wall types, which is in line with WHO recommended estimated time [15]. However, the residual effect of propoxur did not assess for 6 months on standard spray of all wall types and painted wall surface of routine spray as the mortality was $>80 \%$. The residual efficacy of propoxur on smooth and rough wall surface was less than 17 weeks, which was below the WHO recommendation [15]. Therefore, based on this finding, spray cycle may not exceed 17 weeks for routine spray on smooth and rough wall surfaces. The performance of the residual efficacy of propoxur was higher on painted wall surfaces than dung and non-painted mud wall surfaces [9].

Another study by Oxborough and his colleagues reported shorter residual efficacy of deltamethrin on mud wall than concrete against An. arabiensis both in the laboratory and field tests [20]. The study done by Yewhalaw et al. [21] documented higher performance of propoxur on the painted wall type against the laboratory colony of $A n$. arabiensis. On the contrary, maximum persistence of bendiocarb against Anopheles culicifacies was observed on unpainted mud walls, followed by brick and cement walls [22]. However, the residual life of propoxur was little affected by wall types [18]. This difference may be due to difference in chemical nature of the wall surfaces or strains of mosquito species, the $\mathrm{pH}$ of the wall and local temperature or relative humidity.

This study has several strengths. There are limited studies on impact of wall and spray types on residual effect of insecticide at community level in Ethiopia, and the current study may provide evidence to improve vector control. Wild populations of malaria mosquitoes were used for bioassay, which may reflect the actual status in the community as recommended by WHO guidelines. The limitations include the failure to follow the complete WHO recommended duration (up to 6 months) and to conduct quality control test by high performance liquid chromatography due to absence of reagent and dye-functionality of machine. The effect of local temperature, humidity and $\mathrm{pH}$ of the wall surface were not considered in this study as these factors may affect the residual efficacy of propoxur. 


\section{Conclusions}

Wall surface types and spray application quality has an impact on the residual efficacy of propoxur IRS. Propoxur has the highest knockdown and mortality rate of standard spray on all wall surfaces and painted wall surfaces of both spray types. It showed better residual efficacy when applied by strictly following WHO guideline and on painted wall surface types. Therefore, wall surface type available in the community could be considered during decision-making of an IRS programme. IRS could be conducted using national and WHO guidelines to improve residual efficacy of insecticides. Awareness of the importance of painting houses on the residual effect of insecticides could be included in health education programmes. Moreover, research is recommended on impact of spray quality and wall types on residual effect of different insecticides in wide geographic areas.

\section{Abbreviations}

a.i: active ingredient; CS: capsule suspension; EC: emulsifiable concentrate; WG: water-dispersible granule; SC: suspension concentrate.

\section{Authors' contributions}

ZD participated in the planning and implementation of the study, data collection and entry, analyzed the data, drafting the manuscript. FM conceived the study, participated in the planning and implementation and data analysis and, gave critical comment on the draft manuscript. TW participated in the planning the study, and critically commented on the draft manuscript. All authors read and approved the final manuscript.

\section{Acknowledgements}

The authors would like to thank Nigatu Girma for assisting in mosquito collection and field bioassay. We thank the communities who agreed to participate in this field study.

\section{Competing interests}

The authors declare that they have no competing interests.

\section{Availability of data and materials}

The data supporting the conclusions conferred in this article is presented in the main paper.

\section{Consent to publish}

Not applicable.

\section{Ethics approval and consent to participate}

Verbal and written consent was obtained from each household heads to get permission to conduct the study. Information sheets were provided to inform about the purpose of the study, and the inhabitants were informed that involvement in the study was voluntary and had the right to withdraw at any time regardless of reason. Consent was taken from spray men and informed about how to prevent risks during spraying. The trial did not involve any human or animal subjects.

\section{Funding}

This study obtained financial support from Norwegian Programme for Capacity Development in Higher Education and Research for Development (Grant No. NORHED ETH-13/0025). The funding body played no role in study design, field data collection, data analysis and interpretation, and reporting.

\section{Publisher's Note}

Springer Nature remains neutral with regard to jurisdictional claims in published maps and institutional affiliations.

Received: 11 May 2018 Accepted: 14 August 2018

Published online: 20 August 2018

\section{References}

1. WHO. World malaria report 2016. Geneva: World Health Organization; 2016.

2. Bhatt S, Weiss D, Cameron E, Bisanzio D, Mappin B, Dalrymple U, et al. The effect of malaria control on Plasmodium falciparum in Africa between 2000 and 2015. Nature. 2015:526:207-11.

3. WHO. World malaria report 2015. Geneva: World Health Organization; 2015

4. Gish O. Malaria eradication and the selective approach to health care: some lessons from Ethiopia. Int J Health Serv. 1992;22:179-92.

5. Balkew M, Chibsa S, Olana D, Reithinger R, Brogdon W. Insecticide resistance: a challenge to malaria vector control in Ethiopia. Malar J. 2012;11:139.

6. Abraham M, Massebo F, Lindtjorn B. High entomological inoculation rate of malaria vectors in area of high coverage of interventions in southwest Ethiopia: implication for residual malaria transmission. Parasite Epidemiol Control. 2017;2:61-9.

7. WHO. World malaria report 2014. Geneva: World Health Organization; 2014

8. Santos RL, Fayal Ada S, Aguiar AE, Vieira DB, Póvoa MM. Evaluation of the residual effect of pyrethroids on Anopheles in the Brazilian Amazon. Rev Saúde Pública. 2007;41:276-83.

9. WHO. Indoor residual spraying: an operational manual for indoor residual spraying (IRS) for malaria transmission control and elimination. Geneva: World Health Organization; 2015.

10. Etang J, Nwane P, Mbida J, Piameu M, Manga B, Souop D. Variations of insecticide residual bio-efficacy on different types of walls: results from a community-based trial in south Cameroon. Malar J. 2011;10:1186.

11. Bradley J, Matias A, Schwabe C, Vargas D, Monti F, Nseng G, et al. Increased risks of malaria due to limited residual life of insecticide and outdoor biting versus protection by combined use of nets and indoor residual spraying on Bioko Island, Equatorial Guinea. Malar J. 2012;11:242

12. Hailu A, Yewhalaw D, Ambelu A. Evaluation of the efficacy and residual activity of three candidate insecticide formulations for Anopheles gambiae s.I. in Jimma Zone, Southwestern Ethiopia. JNSR. 2016:6:47-50.

13. WHO. Test procedures for insecticide resistance monitoring in malaria vector mosquitoes. Geneva: WHO Pesticide Evaluation Scheme; 2016

14. $\mathrm{FMOH}$. National malaria guidelines. 3rd ed. Addis Ababa: Minister of Health Ethiopia; 2012.

15. WHO. WHO recommended insecticides for indoor residual spraying against malaria vectors. Geneva: World Health Organization; 2015

16. WHO. Guidelines for testing mosquito adulticides for indoor residual spraying and treatment of mosquito nets. Geneva: WHO Pesticide Evaluation Scheme; 2006.

17. Kisinza W, Kabula B, Tungu P, Sindato C, Mweya C, Massue D, et al. Detection and monitoring of insecticide resistance in malaria vectors in Tanzania Mainland. The National Institute for Medical Research Amani Medical Research Center. 2011.

18. PMI. PMI Africa IRS (AIRS) Project Indoor Residual Spraying (IRS 2) Task Order Four, Abt Associates; 2013.

19. Yeebiyo Y, Dengela D, Tesfaye AG, Anshebo GY, Kolyada L, Wirtz R, et al. Short persistence of bendiocarb sprayed on pervious walls and its implication for the indoor residual spray program in Ethiopia. Parasit Vectors. 2016;9:266.

20. Oxborough RM, Kitau J, Jones R, Mosha FW, Rowland MW. Experimental hut and bioassay evaluation of the residual activity of a polymerenhanced suspension concentrate (SC-PE) formulation of deltamethrin for IRS use in the control of Anopheles arabiensis. Parasit Vectors. 2014;7:454. 
21. Yewhalaw D, Balkew M, Shililu J, Suleman S, Getachew A, Ashenbo G, et al. Determination of the residual efficacy of carbamate and organophosphate insecticides used for indoor residual spraying for malaria control in Ethiopia. Malar J. 2012;16:471.
22. Ansari MA, Razdan RK. Impact of residual spraying of bendiocarb against the Malaria vector Anopheles culiicifacies in selected Villages of the Ghaziabad District Uttar Pradesh, India. J Am Mosq Control Assoc. 2004;20:418-23.
Ready to submit your research? Choose BMC and benefit from:

- fast, convenient online submission

- thorough peer review by experienced researchers in your field

- rapid publication on acceptance

- support for research data, including large and complex data types

- gold Open Access which fosters wider collaboration and increased citations

- maximum visibility for your research: over $100 \mathrm{M}$ website views per year

At BMC, research is always in progress.

Learn more biomedcentral.com/submissions 